\title{
O Programa Saúde da Família: contribuições para uma reflexão sobre a inserção do nutricionista na equipe multidisciplinar
}

\section{The Brazilian Family Health Program: contributions to a discussion about the inclusion of the nutritionist in the multidisciplinary team}

Ana Marlúcia Oliveira ASSIS ${ }^{1}$

Sandra Maria Chaves dos SANTOS ${ }^{1}$

Maria do Carmo Soares de FREITAS ${ }^{1}$

Joselina Martins SANTOS ${ }^{1}$

Maria da Conceição Monteiro da SILVA ${ }^{1}$

\section{R E S U M O}

O Brasil experimenta hoje a implementação de um modelo diferenciado para prover atenção básica à saúde, orientado por uma ação multidisciplinar na direção de uma intervenção voltada para a promoção da saúde da população e consubstanciada no Programa Saúde da Família. 0 objetivo de promover a saúde, especialmente no Brasil, não será alcançado sem que ações efetivas na área da vigilância e da assistência alimentar e nutricional sejam implementadas de forma articulada a um sistema de vigilância à saúde. Assim, este trabalho traz à luz algumas reflexões sobre a transição epidemiológica vivenciada no país e situa a problemática alimentar e nutricional nesse processo. Coloca ainda em relevo algumas das proposições básicas do Programa Saúde da Família e da Política Nacional de Alimentação e Nutrição e argumenta como o nutricionista pode contribuir para a necessária transformação, operando nas práticas e nos resultados desse novo modelo de intervenção. Por fim, destaca a impossibilidade de efetivamente transformar a atenção básica à saúde sem o apoio de profissionais que estudam, pesquisam e praticam cotidianamente a vigilância alimentar e nutricional e a assistência dietética e dietoterápica de indivíduos e populações.

Termos de indexação: Programa Saúde da Família, nutricionista, atenção básica à saúde, política publica de saúde.

\footnotetext{
${ }^{1}$ Escola de Nutrição, Universidade Federal da Bahia. R. Araújo Pinho, 32, Canela, 40110-170, Salvador, BA, Brasil. Correspondência para/Correspondenceto: A.M.O.ASSIS.E-mail: amos@ufba.br
} 


\section{A B S T R A C T}

Brazil is presently experiencing the implementation of a differentiated model to provide health basic care, conduced by a multidisciplinary action directed towards an intervention aiming at then population health promotion, consolidated on the Brazilian Family Health Program. The objective of promotion, consolidated on the specially in Brazil, will not be achieved unless effective action in the area of food health monitoring system. Thus, this paper reflects on the epidemiologic transition being experienced in Brazil and discusses the food and nutritional problems in this process. Some basic proposals of Family Health Program and Food and Nutrition National Policy area also emphasized. This article still argues that the nutritionist can contribute yo the necessary transformation, acting on the practices and results of this new model of intervention. Finally, the authors focus on the impossibility of effective transformation of health basic care without the support of professionals who have been studding and practicing the food and nutrition surveillance and the dietary assistance to the populations.

Index terms: family health program, nutritionist, health basic care, public health policy.

\section{N T R O D U Ç Ã O}

Este artigo visa problematizar a ausência do nutricionista nas equipes do Programa Saúde da Família (PSF). Nessa perspectiva, apresenta argumentos que podem auxiliar os profissionais de nutrição a iniciarem uma ampla discussão sobre a sua ausência, mantida, até o presente momento, na implementação de uma proposta que pretende alterar o modelo de prestação de serviços de saúde à população, na direção do fortalecimento das ações de promoção e proteção.

Estas reflexões pretendem alcançar também os responsáveis pela implantação do PSF nas diferentes esferas de governo, enquanto decisores e detentores do poder de favorecer ou obstar o processo de ampliação qualitativa das Equipes de Saúde da Família.

Tendo em vista os objetivos acima referidos, a argumentação deste trabalho desenvolve-se a partir da apresentação de um sucinto cenário sobre a situação de saúde e nutrição da população, o qual sinaliza para a importância que os saberes e práticas em alimentação e nutrição vêm adquirindo na conquista e manutenção de uma melhor qualidade de vida para todos os brasileiros.
$\mathrm{Na}$ continuidade, são apresentados aspectos centrais das respostas governamentais aos problemas sinalizados na seção anterior, abordando o significado do PSF, enquanto uma modalidade diferente de prestar serviços de saúde. A seguir são evidenciadas diretrizes e prioridades da Política Nacional de Alimentação e Nutrição (PNAN) enquanto integrante da Política Nacional de Saúde, constituindo componente básico do Sistema Único de Saúde (SUS). Nesta seção buscase argumentar que a realização plena dos objetivos do PSF guarda estreita dependência com uma maior capacidade de intervenção na área da alimentação e nutrição, reconhecendo-se na estratégia deste Programa uma oportunidade ímpar para avanços na conquista da segurança alimentar e nutricional dos brasileiros.

A partir dessa contextualização, na seqüência são sistematizados os argumentos julgados relevantes para subsidiar a defesa da inserção do Nutricionista na equipe do PSF, pondo em relevo sua formação e 0 arsenal de práticas que detém, assim como o seu compromisso social com a melhoria do estado de saúde e nutrição da população.

Enfim, parafraseando Darcy Ribeiro, as autoras reafirmam com este trabalho serem profissionais da academia, pesquisadoras e responsáveis pela formação de Nutricionistas na 
graduação e na pós-graduação, e com estas reflexões desejam apresentar uma contribuição acadêmica “...que aspira a influir sobre as pessoas, que aspira a ajudar o Brasil a encontrar-se a si mesmo" (Ribeiro, 1995).

\section{A SA Ú DE E A N U T IC Ã O DA FA M ÍlIA BRASILEIRA}

A transição epidemiológica em curso no Brasil, fruto das mudanças no perfil demográfico e nutricional da população, vem promovendo profundas alterações no padrão de morbi-mortalidade e no estado nutricional em todas as faixas etárias, em todo o espaço geográfico, nas áreas urbana e rural (M onteiro, 1995; Gigante et al., 1997; Assis et al., 2000a) das grandes e pequenas cidades.

Embora o perfil de saúde da população brasileira possa ser caracterizado por decrescentes taxas de mortalidade em todas as faixas etárias (Barreto \& Carmo, 1994), destacam-se ainda as altas prevalência e incidência de várias doenças infecciosas e parasitárias (Brasil, 1999b), de elevada ocorrência em países periféricos, somando-se a esse panorama aquelas morbidades características dos países centrais. De uma maneira geral, o quadro de morbi-mortalidade desenhado na atualidade para o Brasil associa-se com a alimentação, a nutrição e o estilo de vida dos brasileiros (World Health..., 1997).

Observa-se, por outro lado, um rápido crescimento da população de idosos (indivíduo com idade superior a 60 anos), indicando que o processo de envelhecimento está em curso no país, e com ele, a ascensão das doenças não transmissíveis. Entre os adultos destaca-se a elevação das taxas de doenças como diabetes, obesidade, neoplasia, hipertensão arterial e hiperlipidemias (Barreto \& Carmo, 1994), característica semelhante ao perfil de morbi-mortalidade de população adulta de países desenvolvidos (Gigante et al., 1997; Organização Panamericana..., 1998).
Entre os adolescentes, a obesidade, a hipertensão arterial e as hiperlipedemias já acenam também como problemas importantes de saúde e se mostram fortemente associadas às condições de nutrição e ao estilo de vida adotado e/ou imposto pela sociedade moderna (World Health..., 1997; Fonseca et al., 1998).

0 perfil da morbi-mortalidade na população pré-escolar caracteriza-se por expressiva redução da severidade das doenças infecciosas e parasitárias, embora as prevalências continuem altas. As formas grave e moderada da desnutrição energético-protéica entraram em declínio, mas persistem as elevadas freqüências da forma leve (Pesquisa Nacional..., 1996), observando-se o despontar do problema do sobrepeso e da obesidade neste grupo populacional (Pesquisa Nacional..., 1996; Assis et al., 2000a). Associam-se a esse quadro as deficiências específicas de micronutrientes, com altas prevalências, particularmente da vitamina A (Santos et al., 1996a; Santos et al., 1996b; Assis et al., 1997; Instituto Nacional..., 1998) e do ferro dietético, repercutindo em elevadas taxas de anemia (Assis et al., 1997; Instituto Nacional..., 1998; Assis et al., 2000a) e acometendo principalmente as crianças pré-escolares, especialmente aquelas que vivem na Região Nordeste. Assim, para esse grupo populacional o padrão da morbi-mortalidade está também associado direta ou indiretamente às condições de alimentação e nutrição.

É importante comentar sobre o perfil de morbidade da gestante, caracterizado também pela dualidade do estado de saúde e nutrição, dividindo-se entre aquele representado pelo peso inadequado ao gestar e o baixo ganho de peso no processo gestacional, resultando em baixo peso ao nascer e suas conseqüências negativas para a sobrevivência do recém-nascido, e aquele caracterizado pelo sobrepeso, pelas doenças cardiovasculares pelo diabetes e pela obesidade, os quais constituem riscos elevados de morbidade materna (World Health, 1995). Juntam-se ainda a esse quadro as deficiências específicas de 
micronutrientes, particularmente de ferro, redundando em elevadas taxas de anemia (Assis et al., 2000b), carência reconhecidamente associada ao baixo peso ao nascer.

Nesse cenário, o Brasil, ao colocar-se como signatário dos Fóruns Internacionais sobre Segurança Alimentar, assume os objetivos estratégicos da redução das prevalências da anemia ferropriva, da hipovitaminose A (Food..., 1992), da deficiência de iodo, do baixo peso ao nascer e de outras deficiências nutricionais as quais vêm comprometendo a qualidade de vida e a saúde do brasileiro.

Ressalta-se, no entanto, que a falta de equidade em saúde e a distribuição heterogênea dos seus agravos permeiam todas as instâncias e todos os ciclos de vida da população brasileira, definindo-se nessa complexidade social o perfil nutricional e alimentar diretamente vinculado ao padrão de morbi-mortalidade desta comunidade.

Diante dessa situação, o Brasil vem buscando, especificamente a partir dos anos 90s, reorganizar o modelo de atenção à saúde, de forma a superar a história de um sistema nacional de saúde modelado pela assistência médica curativa, de baixa resolutividade e inacessível à maioria da população.

\section{RE CENTES RESPOSTAS G O VERNAM ENTAIS AOS PRO BLEMAS DE SA Ú DE E N UTRIÇÃ 0: 0 PSF E P N A N}

\section{Reformando o modelo assistencial: a estratégia Programa Saúde da Família}

A partir dos anos 80s, sob o cenário da redemocratização do país, emergiu o M ovimento Sanitário, o qual possibilitou a construção gradativa de uma reforma que se iniciou em 1986, com as Ações Integradas de Saúde (AIS), passou pelo Sistema Unificado e Descentralizado de Saúde (SUDS) em 1987 e alcançou o Sistema Único de Saúde (SUS) em 1988, de existência e princípios garantidos constitucionalmente. Embora a temporalidade possa indicar a rapidez desse processo, a recuperação histórica assinala a rede de conflitos de interesses e os obstáculos diversos que permearam este movimento (Viana e Castro, 1993).

O Programa Saúde da Família surgiu em 1994, sob a égide da discussão da reformulação do sistema de saúde, a qual ampliou a concepção de atenção, primeiramente definida na Norma Operacional Básica do Sistema Único de Saúde (NOB/SUS 1/93) e posteriormente reorganizada pelas Normas da NOB/SUS 1/96. O PSF teve como precursor o Programa de Agentes Comunitários de Saúde (PACS), implantado em 1991, e adotou como referência as experiências internacionais e nacionais de extensão da cobertura à saúde por meio de assistência às famílias no próprio domicílio em articulação com as unidades de saúde (Brasil, 2001).

O PSF foi então ancorado nos pressupostos da prevenção e não no processo curativo em si. Tais pressupostos, baseados em uma visão ampla, definiram que a Atenção Básica deve compreender um " conjunto de ações de caráter individual ou coletivo, situada no primeiro nível de atenção dos sistemas de saúde voltada para a promoção da saúde, prevenção de agravos, o tratamento e a reabilitação" (Brasil, 2000a).

A compreensão subjacente ao Programa Saúde da Família colocou em relevo a intervenção multidisciplinar sobre os agravos à saúde da população. Neste sentido, o Departamento de Atenção Básica (DAB) passou a reunir diferentes áreas de atuação - Dermatologia Sanitária, Controle do Diabetes Mellitus, Controle da Hipertensão, Saúde Bucal, Assistência Farmacêutica Básica, Pneumologia Sanitária e Área Técnica de Alimentação e Nutrição, tendo por pressuposto que essas áreas “ irão contribuir de forma significativa para a concepção e sistematização de todas as ações relativas à atenção básica e no processo de consolidação do PACS/PSF" (Brasil, 2000b). 
Desse modo, o Programa Saúde da Família representa a estratégia de reorientação do modelo de atenção básica à saúde, e, assim, pode ser entendido como uma nova etapa nessa busca de fazer a saúde de forma diferente, sendo considerado eficiente, eficaz e capaz de aproximar os serviços, os profissionais de saúde e a população, além disso o PSF vem apresentado como detentor do propósito de "reorganizar a prática da atenção à saúde em novas bases e substituir o modelo tradicional, levando a saúde para mais perto da família e, com isso, melhorar a qualidade de vida dos brasileiros", propondo-se a resolver $85 \%$ dos problemas de saúde da população da área de abrangência do programa (Brasil, 2001).

Portanto, o Programa Saúde da Família pode ser caracterizado como uma estratégia central na reestruturação do sistema no que se refere à organização da atenção primária, "a partir de um conjunto de ações conjugadas em sintonia com os princípios de territorialização, intersetorialidade, descentralização, co-responsabilização e priorização de grupos populacionais com maior risco de adoecer e morrer" (Trad \& Bastos, 1998).

Para tanto, o PSF atua a partir da organização de Equipes de Saúde da Família (ESF), integradas idealmente por um médico, um enfermeiro, um auxiliar de enfermagem e quatro a seis Agentes Comunitários de Saúde (ACS)², embora se pressuponha que outros profissionais possam ser incorporados de acordo com a demanda dos serviços (Brasil, 2000a). Cada equipe se responsabiliza pela atenção a grupos de seiscentas a mil famílias, tendo como limite máximo o total de 4500 indivíduos, cadastrados a partir de levantamento domiciliar (Machado, 2000). A estas equipes são colocados os desafios de compreender melhor o processo saúde/doença experimentado pela população de sua área de atuação e também a tarefa de ir além da prática curativa tradicional (M achado, 2000).
O PSF experimentou um crescimento vertiginoso em pouco espaço de tempo, em parte pelas estratégias de indução adotadas pelo Governo Federal, de tal forma que, entre 1994 e 2000, a quantidade de ESF aumentou de 328 para 9 664, atuando em 2900 municípios da Federação ${ }^{3}$. Os números sobre cobertura registram, para 2000, um total de trinta e três milhões de pessoas acompanhadas (Machado, 2000) e, em maio de 1999, os dados oficiais informam a implantação do PSF em 1735 dos 1787 municípios do Nordeste do país (Brasil, 2001).

Os números e os perfis dos recursos humanos envolvidos no PSF interessam de perto à discussão deste trabalho. Em dezembro de 1998 atuavam no Programa, em todo o país, 6294 profissionais das áreas da medicina e da enfermagem ( $50 \%$ de cada categoria). Em junho de 1999 este valor foi reduzido para 5264 profissionais, consistindo em 2703 médicos $(51,3 \%$ ) e 2561 enfermeiros (48,7\%) (Machado, 2000).

Dados da avaliação da implantação do Programa realizada em 1999, a partir das informações dos gestores locais de saúde, revelam que a determinação da composição mínima das equipes vinha sendo cumprida na maioria dos municípios, sendo o médico, o enfermeiro e o agente comunitário de saúde os componentes de aproximadamente $96,0 \%$ delas, com leve predomínio do médico, o qual compõe a equipe em 98,2\% dos municípios estudados (Brasil, 1999a). Observa-se, ainda, que a participação de outros profissionais da saúde nas ESF é reduzida. O odontólogo faz parte delas em $28,8 \%$ dos municípios e o assistente social em 9,3\%; a presença do psicólogo foi identificada em 5,3\% das equipes e o nutricionista foi citado como seu membro em 4,5\% dos casos (Brasil, 1999a).

20 número de ACS nas equipes pode variar de acordo com o número de famílias acompanhadas. A proporção sugerida é de um agente para cada 575 pessoas.

${ }^{3}$ Até 1998 os estados do Amapá e Roraima eram os únicos sem equipes do programa, segundo dados da pesquisa coordenada por Maria Helena Machado (2000). 
As equipes implantadas vêm atuando nas mais diversas áreas, lidando com múltiplos problemas de saúde dos indivíduos em diferentes momentos fisiológicos. De acordo com dados do M inistério da Saúde, 70\% delas encontravam-se, em 1999, realizando concomitantemente atividades relativas a oito grandes programas prioritários, envolvendo o pré-natal, a assistência à criança, o controle da hipertensão, do diabetes, das doenças sexualmente transmissíveis (DST), da tuberculose e da hanseníase, e ações de vigilância epidemiológica. A realização de ações na área do saneamento básico foi referida por cerca de $70 \%$ das equipes que integraram a pesquisa e um menor percentual (35\% ) referiu realizar ações de vigilância sanitária, incluindo vigilância de medicamentos, de alimentos e da qualidade da água e também ações educativas (Brasil, 1999b).

A natureza transdisciplinar do conjunto de tarefas previstas para a ESF, assim como a natureza multifacetada dos problemas a serem enfrentados e o desafio de fazer avançar o modelo na direção da integralidade da atenção, podem representar algumas das razões as quais levam os profissionais integrantes da equipe a considerar o trabalho como "muito desgastante" $(68,4 \%$ dos médicose $59,6 \%$ dos enfermeiros). Nessa mesma direção, registra-se que $96,5 \%$ dos médicos e $96,9 \%$ dos enfermeiros demandaram maiores oportunidades de aprimoramento, de forma a fazer face aos desafios definidos pelo Programa (Machado, 2000).

Embora, segundo as avaliações realizadas até o momento, a composição mínima das ESF venha produzindo excelentes resultados em termos de extensão da cobertura, com efeitos positivos sobre a redução das taxas dos indicadores de morbi-mortalidade, ela apresenta limites técnicos e físicos, diante da necessidade de fazer uma saúde diferente, isto é, capaz de estender a prática clínica para fora dos muros da unidade de saúde e prestar uma atenção integral à saúde da população em consonância com os princípios norteadores de sua criação.
A integralidade da atenção é um princípio básico nessa discussão sobre as condições necessárias para a construção de um novo modelo assistencial. 0 art. 198, Seção II, da Constituição Brasileira estabelece 0 atendimento integral à saúde como uma das diretrizes do SUS, destacando a prioridade das atividades preventivas, sem prejuízo das assistenciais, devendo tal preceito ser aplicado em todos os níveis de atenção, implicando na compreensão do indivíduo como singular e como parte de uma coletividade.

A partir daí, admite-se que a atenção integral, conforme preconizada, só se concretiza através da atuação de uma equipe multidisciplinar, pois a mudança nas formas de intervir é decorrente de uma " atuação e abordagem interdisciplinar ao indivíduo e à população na realidade em que se inserem" (Carvalho, 1993). Além disso, a " interdisciplinaridade caracteriza-se pela intensidade das trocas entre os especialistas e pelo grau de integração real das disciplinas" (Carvalho, 1993).

Como componente do trabalho interdisciplinar, é oportuno destacar as ações de alimentação e nutrição em quaisquer formas de intervenção, como estratégias indispensáveis a todo programa cuja finalidade seja elevar a qualidade de vida da população a partir do princípio da integralidade. Neste sentido, importa ressaltar os aspectos específicos sobre o perfil dos profissionais que compõem a equipe do PSF em relação à prestação da atenção particularmente voltada para o campo da alimentação e nutrição.

Até 1999, apenas dois médicos de um conjunto de 550 que responderam ao item específico de uma pesquisa promovida pelo MS informaram deter titulação de especialistas na área da nutrologia, e nenhum dos entrevistados referiu atuar neste ramo. As áreas predominantes de atuação dos médicos foram a medicina interna $(28,4 \%)$, seguida da medicina comunitária $(14,4 \%)$ e da pediatria $(13,4 \%)$ (Machado, 2000$)$.

Entre os enfermeiros, cerca de $22,0 \%$ informaram atuação em saúde pública, $11,7 \%$ 
na unidade de saúde, 11,1\% em gerenciamento de serviços de enfermagem e 10,9\% em enfermagem médico-cirúrgica. No estudo realizado, apenas $2,7 \%$ destes profis-sionais indicaram atuar na área de enfermagem maternoinfantil e $1,8 \%$ em enfermagem pediátrica (Machado, 2000).

Destaca-se ainda que, no Brasil, tanto 0 médico como o enfermeiro, em virtude do seu currículo, recebem pouca ou nenhuma informação em nutrição no curso da graduação. A Pós-Graduação em saúde pública ou na área materno-infantil poderia corrigir parcialmente esta ausência. No entanto, até 1999, menos de $40 \%$ dos profissionais atuantes nas equipes haviam realizado algum curso de especialização na área. Percentual ainda menor (menos de 3\%) havia cursado Pós-Graduação em nível de mestrado ou doutorado (Machado, 2000).

Assim, pode-se questionar se a equipe mínima proposta para o PSF conseguirá em médio prazo avançar na integralidade da atenção. Focalizando particularmente a área da alimentação e nutrição, pode-se pressupor que se encontram ausentes na equipe, hoje formada, condições técnicas e operacionais para inovar na atenção, em direção à segurança alimentar. $A$ apresentação das diretrizes e prioridades da Política Nacional de Alimentação e Nutrição (PNAN), na continuidade, subsidia a afirmação precedente.

\section{Promoção e proteção à saúde com segurança alimentar: a Política Nacional de Alimentação e Nutrição}

No Brasil os problemas de alimentação e nutrição da população vêm sendo alvo de interven ções governamentais desde os anos 40s, mas foi apenas a partir da década de 70 que esse campo passou a conformar uma política de âmbito nacional, consubstanciada no Programa Nacional de Alimentação e Nutrição (PRONAN), criado em 1976 pelo extinto Instituto Nacional de Alimentação e Nutrição (INAN), vinculado ao M inistério da Saúde (Santos, 2001). Desde então, também se construiu a história de uma forma de intervenção centrada no assistencialismo, via programas parciais, ineficientes e descontínuos de suplementação alimentar, voltados para indivíduos, particularmente para os trabalhadores e para os integrantes dos chamados grupos de risco, como as gestantes, lactentes e pré-escolares. Registra-se, ainda, que esses programas primavam por manter interfaces pouco nítidas com a política de saúde em vigor.

A exemplo da saúde como um todo, também a área específica da alimentação e nutrição teve seus programas revisitados e avaliados durante o processo de transição democrática, gerando propostas para a inclusão de um capítulo específico na Constituição Brasileira para declarar o direito de todos à alimentação. Tal intento não foi alcançado, mas o enfrentamento da fome/desnutrição foi repolitizado, inclusive adotando-se o conceito de segurança alimentar como norteador das ações nesse domínio.

Neste cenário, é oportuno comentar que - Brasil assumiu compromissos internacionais e nacionais em relação à Segurança Alimentar e Nutricional da população por ocasião da Cúpula Mundial de Roma (CUMBRE) (Food..., 1996), devendo, portanto, fomentar as estratégias necessárias para honrar estas obrigações. A segurança alimentar e nutricional, como modernamente vem sendo concebida, implica a garantia do acesso de todos, em todos os lugares, aos alimentos que atendam às suas necessidades nutricionais e correspondam às suas preferências, não só eliminando as mazelas da fome, mas também provendo a criação de um ambiente social e sanitário capaz de assegurar a todos o melhor aproveitamento dos alimentos consumidos e, conseqüentemente, a melhoria do estado de saúde e da qualidade de vida (Food..., 1996).

Neste contexto pode-se explicar a atual Política Nacional de Alimentação e Nutrição (PNAN), proposta para ser implementada pelo setor da saúde como parte integrante da Política Nacional de Saúde, cujo propósito é "a garantia da qualidade dos alimentos colocados para o 
consumo no país, a promoção de práticas alimentares saudáveis e a prevenção e o controle dos distúrbios nutricionais", entendendo-se caber ao Estado e à sociedade respeitar, proteger e facilitar ações para permitir a todos se alimentarem de forma digna e indicando que a realização plena da segurança alimentar extrapola a área da saúde, exigindo uma intervenção intersetorial (Brasil, 2000d).

Considerando as responsabilidades do setor da saúde neste processo, a PNAN elenca um conjunto de diretrizes as quais resultam em ações de diferentes naturezas (Quadro 1). A partir da análise deste quadro, evidencia-se a complexidade das tarefas percebidas como necessárias para uma intervenção qualificada do setor da saúde sobre os problemas de alimentação e nutrição. Nota-se também que estas diretrizes são materializadas em um conjunto de ações específicas no campo da promoção da saúde e da nutrição e na prevenção das doenças, cuja operacionalização está associada ao cumprimento da agenda da alimentação saudável, inserida no contexto da reorientação da atenção básica de saúde (Brasil, 2000d). Além disso, a unidade familiar, alvo do Programa Saúde da Família, é também a prioridade da intervenção em nutrição.

A avaliação da implantação do PSF indica que, em 1999, as equipes deste Programa vinham executando várias ações próprias da área da nutrição, além de promover uma extraordinária ampliação do atendimento (Brasil, 1999a). 0

Quadro 1. Principais diretrizes e ações previstas na Política Nacional de Alimentação e Nutrição. Brasil, 1999.

\begin{tabular}{|c|c|c|}
\hline Diretrizes & Detalhamento & Ações previstas \\
\hline $\begin{array}{l}\text { Estímulo às ações inter-setorias que propiciem } \\
0 \text { acesso universal aos alimentos. }\end{array}$ & $\begin{array}{l}\text { Responsabilidade do setor da saúde na } \\
\text { identificação e divulgação dos determinantes } \\
\text { da situação alimentar e nutricional. }\end{array}$ & $\begin{array}{l}\text { Produção de informações, divulgação e negociação com } \\
\text { outros setores governamentais que controlam políticas } \\
\text { de interesse para a redução do risco de insegurança } \\
\text { alimentar. }\end{array}$ \\
\hline $\begin{array}{l}\text { Monitoramento da situação alimentar e } \\
\text { nutricional. }\end{array}$ & $\begin{array}{l}\text { Descrição contínua e predição de tendências } \\
\text { das condições de alimentação e nutrição da } \\
\text { população e de seus determinantes como } \\
\text { parte da rotina da atenção básica. }\end{array}$ & $\begin{array}{l}\text { Ampliação e aperfeiçoamento do Sistema de Vigilância } \\
\text { Alimentar e Nutricional - SISVAN, contemplando, entre } \\
\text { outros atos: diagnóstico descritivo e analítico dos } \\
\text { principais problemas de saúde, instalação de sítios } \\
\text { sentinelas em áreas de maior risco, mapeamento das } \\
\text { endemias carenciais, mapeamento da prática do } \\
\text { aleitamento materno, avaliação periódica do estado } \\
\text { nutricional dos escolares, levantamento de dados } \\
\text { macroeconômicos e sociais que definem riscos da } \\
\text { insegurança alimentar e nutricional. }\end{array}$ \\
\hline $\begin{array}{l}\text { Promoção de práticas alimentares e estilos de } \\
\text { vida saudáveis. }\end{array}$ & $\begin{array}{l}\text { Estratégias para estimular e ampliar a adoção } \\
\text { pela população de práticas alimentares } \\
\text { saudáveis como parte essencial do objetivo } \\
\text { de promover estilos de vida saudáveis para } \\
\text { todos. }\end{array}$ & $\begin{array}{l}\text { Socialização permanente e metodologicamente } \\
\text { renovada de conhecimentos sobre os alimentos e o } \\
\text { processo de alimentação, abrangendo o resgate de } \\
\text { hábitos e práticas alimentares regionais que impliquem } \\
\text { em consumo de alimentos de baixo custo e alto valor } \\
\text { nutritivo, a aplicação de fatores nutricionais na } \\
\text { prevenção e recuperação de doenças crônicas, a adoção } \\
\text { de medidas para disciplinamento da publicidade de } \\
\text { produtos alimentícios, o fortalecimento dos bancos de } \\
\text { leite humano. }\end{array}$ \\
\hline $\begin{array}{l}\text { Prevenção e controle dos distúrbios } \\
\text { nutricionais e das doenças associadas à } \\
\text { alimentação e nutrição. }\end{array}$ & $\begin{array}{l}\text { Dupla perspectiva de ação, considerando o } \\
\text { quadro da morbi-mortalidade infantil } \\
\text { dominado pelo binômio desnutrição/infecção } \\
\text { e o grupo das enfermidades crônicas não- } \\
\text { transmissíveis. }\end{array}$ & $\begin{array}{l}\text { Prevenção e controle dos distúrbios nutricionais, } \\
\text { incluindo, entre outras atividades: abordagem familiar } \\
\text { via adoção do conceito de "família vulnerável", ações } \\
\text { de suplementação alimentar, educação alimentar, } \\
\text { vigilância do crescimento e desenvolvimento das } \\
\text { crianças e de outros membros da unidade familiar, } \\
\text { controle de doenças coexistentes, suplementação } \\
\text { específica (ferro e retinol), fortificação de alimentos, } \\
\text { fiscalização da iodação do sal, ações de estímulo ao } \\
\text { aleitamento materno. }\end{array}$ \\
\hline
\end{tabular}

Fonte: Brasil (1999a). 
aperfeiçoamento desta atuação é um imperativo. Como apresentado e argumentado na seção seguinte deste trabalho, o nutricionista brasileiro vem adquirindo, ao longo dos anos, os conhecimentos para atuar neste campo, sendo o profissional que se coloca em condições de integrar a equipe do PSF e promover o encontro entre este Programa e a PNAN, em beneficio da situação de saúde, alimentação e nutrição da população.

\section{O PROGRA M A SA Ú DE DA FAM ÍLIA, A NUTRIÇÃ O E N U TRICIONISTA}

Tomando como base os fundamentos norteadores das diretrizes da Política Nacional de Alimentação e Nutrição, pode-se concluir que a efetivação da vigilância capaz de ultrapassar a abordagem do estado nutricional, abrangendo a dimensão alimentar, demanda também assegurar a disponibilidade e as condições de acesso social e físico aos alimentos. Se, por um lado, esta questão está estritamente ligada às condições estruturais da sociedade e requer estudos para minimizar a problemática, por outro lado, é imperioso conhecer com profundidade os determinantes culturais dos hábitos alimentares, assim como compreender porque práticas alimentares regionais saudáveis vêm sendo substituídas por outras que agregam maiores prejuízos à saúde e, por vezes, também às finanças das famílias. Todo esse desafio é emoldurado, no âmbito da PNAN, pela educação alimentar e nutricional, a qual exige um profissional detentor de conhecimentos no campo da ciência da alimentação e da nutrição que, conseqüentemente, integre à sua prática ferramentas da epidemiologia, do planejamento, da educação e de outras disciplinas das ciências sociais e humanas.

Além disso, o processo de adoecimento da população é explicado por uma rede complexa de determinantes biológicos, sociais, econômicos e culturais de difícil hierarquia na definição da situação de risco e de suas prioridades. Desse modo, a prevenção e o tratamento dos males que atingem os brasileiros requerem a disponibilidade de uma equipe multidisciplinar, capaz de conceber a saúde na sua dimensão coletiva. Os saberes e as práticas associados, e não divorciados, permitirão o exame minudente destas enfermidades, assegurando-se, no entanto, a prática dos saberes específicos, enquanto campos de competências necessários para a resolução dos problemas de saúde da população brasileira.

Neste sentido, toma-se como referência o discurso oficial do PSF:

"O Programa Saúde da Família não é Programa do M édico da Família, pois não é centrado no trabalho médico, mas de uma equipe multiprofissional, na qual todos os saberes são respeitados..." (Brasil..., 2000c).

Tal afirmação, perfeitamente sustentada na compreensão da multideterminação dos problemas de saúde vivenciados pela população e amparada no conceito da interdisciplinaridade, evidencia que a implementação do programa prevê o envolvimento de profissionais qualificados, capazes de articularem no exercício de seu trabalho os conhecimentos específicos, construídos ao longo de sua formação profissional, com os saberes coletivos, na direção de uma prática social a qual transcenda à fragmentação e especialização características das ações de saúde no País.

$\mathrm{Na}$ atualidade, de acordo com $\mathrm{o}$ conhecimento científico acumulado, o controle, a prevenção e o tratamento das enfermidades aqui aludidas passam, necessariamente, pelo campo específico da ciência da nutrição, assim como as mudanças na qualidade de vida das pessoas, as quais são preconizadas em todo o mundo como uma das estratégias capazes de gerar impactos positivos no perfil epidemiológico das populações.

A importância do componente alimentar e nutricional nas políticas públicas de corte social, particularmente neste país, é mais um indicador da relevância dessa área de saberes e práticas. Desde os anos 70s o Brasil vem buscando, em 
nível nacional, implementar programas de intervenção na área de alimentação e nutrição. Mais recentemente tais ações passaram a ser descentralizadas. No presente, tanto quanto no passado, questões sobre avaliação do impacto dessas políticas e programas encontram-se na agenda do governo, não só pela inversão de recursos que representam, mas também pelo compromisso firmado por todos os governos e governantes com a redução da fome e da prevalência da desnutrição do povo brasileiro.

A competência do nutricionista para integrar a equipe do Programa Saúde da Família está estabelecida em sua formação acadêmica, a qual o instrumentaliza a realizar o diagnóstico nutricional da população, tornando-o, assim, o único profissional a receber uma instrução específica que lhe permite, a partir desse diagnóstico e da observação dos valores socioculturais, propor orientações dietéticas cabíveis e necessárias, adequando-as aos hábitos da unidade familiar, à cultura, às condições fisiológicas dos grupos e à disponibilidade de alimentos. Trata-se, portanto, de um profissional apto a participar efetivamente da recriação das práticas de atenção à saúde no Brasil. Por este caminho, pode-se afirmar que uma assistência à saúde da família brasileira, cujo objetivo seja transformar a história das práticas e dos resultados das intervenções, não poderá prescindir da atuação do nutricionista.

A partir da integração do nutricionista à equipe do Programa Saúde da Família, estará conquistada a sua oportunidade de colocar à disposição da unidade familiar, de forma integrada com outros profissionais, os seus saberes específicos na direção de uma ação responsável sobre os problemas que afetam a saúde e a qualidade de vida da população brasileira.

\section{O NSIDERAÇÕ ES FINA IS}

A partir dos elementos apresentados, os quais consideram o cenário da transição demográfica e epidemiológica experimentado pelo Brasil e a relevância da situação alimentar e nutricional da população, destacando também a reforma da atenção básica da saúde adotada no país, materializada no Programa Saúde da Família, que prioriza a forma integral de fazer saúde, argumenta-se a favor da inserção do nutricionista na equipe deste Programa.

Questiona-se ainda quais os rumos possíveis de uma reforma da atenção básica à saúde sem a integração da nutrição e do nutricionista em sua prática. Se o saber específico deste profissional não é verdadeiramente contemplado nesta mudança, tem-se a impressão de que essa questão não está na esfera do pragmático, mas é simplesmente filosófica - não compreendendo o verdadeiro "estado da arte de fazer saúde". Se assim for, o novo modelo continuará sendo centrado na velha hegemonia do conhecimento médico, bloqueando o processo de democratização dos espaços profissionais e fortalecendo a idéia, mais uma vez, do predomínio de uma percepção simplista da complexidade dos problemas vivenciados pela população para a qual esse Programa foi inicialmente direcionado.

A não exclusão do nutricionista da equipe mínima do Programa Saúde da Família cerceia a própria sociedade de vir a utilizar os saberes por ela propiciados a esses profissionais, negando-Ihe o direito concedido por Lei, e faz supor que mais uma vez o país vai conviver com do is modelos de atenção à saúde: um constituído pelo Programa Saúde da Família e possivelmente um outro remanescente do sistema o qual será mantido nas Unidades de Saúde.

Baseado na compreensão da filosofia do Programa, cuja função norteia em estratégia estruturante e provocadora de modificações internas e capazes de romper com a dicotomia entre a atuação da saúde pública e a atenção médica na direção de um novo modelo de reorganização das ações e serviços de saúde, percebe-se a necessidade de integrá-lo a outros saberes e outras competências. Nesse sentido, este documento fundamenta a necessária discussão sobre a participação do nutricionista 
neste projeto de transformação da história da saúde no país, ao lado de outros profissionais da área, com os quais já dialoga nos espaços da clínica hospitalar e ambulatorial.

Assim, pretende-se que seja referendado o papel do nutricionista na sociedade global e no setor da saúde em particular, não só pelo prisma da sua identidade profissional, como detentor do saber científico na área da alimentação e nutrição, mas também pela destreza de o colocar em benefício de outrem, e assim contribuir com a melhoria da qualidade de vida da população brasileira.

\section{REFERÊ NCIAS B I B LIO G RÁ F I C A S}

ASSIS, A.M.O., SANTOS, L.M.P., MARTINS, M.C., ARAÚJO, M.P.N., AMORIN, D.Q., MORRIS, S.S., BARRETO, M.L. Distribuição da anemia em pré-escolares do semi-árido da Bahia. Cadernos de Saúde Pública, Rio de Janeiro, v.13, n.2, p.237-243, 1997.

ASSIS, A.M.O, BARRETO, M.L., SANTOS, L.M.P., SAMPAIO, L.R., MAGALHÃES, L.P., PRADO, M.S., SANTOS, N.S., GALVÃO, N.M., SILVA, R.C., OLIVEIRA, V.A. Condições de vida, saúde e nutrição na infância em Salvador. Salvador : Bureau, 2000a. 163p.

ASSIS, A.M.O., CARVALHO, G., PONTES, A. Distribuição da anemia materna e em seus recém-nascidos: relatório preliminar. Salvador: M inistério da Saúde, 2000b. 21p.

BARRETO, M.L., CARMO, E.H. Situação de saúde da população brasileira: tendências históricas, determinantes e implicações para as políticas de saúde. Informe Epidemiológico. Brasil. Ministério da Saúde, Fundação Nacional de Saúde. Brasília (DF), v.3, p.5-32, 1994.

BRASIL. M inistério da Saúde. Secretaria de Assistência à saúde/CAB. Avaliação da implantação e funcionamento do Programa Saúde da Família-PSF: relatório preliminar 1999a. [online]. Disponível em: <http://www gov.Br/psf/programa/publicações htm>. Acesso em: 20 Jul. 2001.
BRASIL. M inistério da Saúde. Fundação Nacional de Saúde. Informe Epidemiológico do SUS. Evolução temporal das doenças de notificação compulsória no Brasil de 1980 a 1998. Brasília, 1999b. 43p.

BRASIL. M inistério da Saúde. Anais da reunião técnica dos pólos de capacitação, formação e educação permanente em saúde da família, Brasil. Brasília, 2000a. 84p.

BRASIL. M inistério da Saúde. Secretaria de Políticas de Saúde. Brasil. Brasília, 2000b. p.34.

BRASIL. Ministério da Saúde. Abrindo a porta para dona saúde entrar: uma estratégia para a reorganização do modelo assistencial, Brasil. Brasília, 2000c. 19p.

BRASIL. M inistério da Saúde. Políticas de alimentação e nutrição, área técnica de alimentação e nutrição, Brasil. Brasília, 2000d. p.48.

BRASIL. M inistério da Saúde. Alimentação e nutrição: estado nutricional da clientela da Saúde da Família e do ICCN da Região Nordeste. Informe da Atenção Básica, Brasília, v.2, n.8, p.1-2, 2001.

CARVALHO, M.R. Modelos assistenciais de unidades básicas e o conceito de integralidade: In: Bodstein, R.C.A. (Org.). Serviços locais de saúde: construção de atores e políticas. Rio de Janeiro : Relume-Dumará, 1993. p.118-130.

FONSECA, V.M., SICHIERI, R., VEIGA, G.V. Fatores associados à obesidade em adolescentes. Revista de Saúde Pública, São Paulo, v.32, 1998 [online]. Disponível em: <http://www.scielo.br/rsp>. Acesso em 20 jul. 2000.

FOOD AGRICULTURE ORGANIZATION. International conference on nutrition: final report of the conference. Rome, 1992. 55p.

FOOD AGRICULTURE ORGANIZATION. Declaración de Roma sobre la seguridad alimentaria mundial y plan de acción de la Cumbre mundial sobre la Alimentación. Roma, 1996. p.48

GIGANTE, D.P., BARROS, F.C., POST, C.L.A., OLINTO, $M$.T.A. Prevalência da obesidade em adultos e seus fatores de risco. Revista de Saúde Pública, São Paulo, v.31, 1997. [online]. Disponível em: <http:// www.scielo.br/rsp>. Acesso em 20 Jul. 2000. 
INSTITUTO NACIONAL DE ALIMENTAÇÃO E NUTRIÇÃO. INAM/MS, II Pesquisa estadual de saúde e nutrição: Saúde, nutrição, alimentação e condições socioeconômicas no Estado de Pernambuco: relatório. Brasília : Ministério da Saúde, 1998. 117p.

MACHADO, M.H. (Coord.). Perfil dos médicos e enfermeiros do Programa Saúde da Família no Brasil: relatório final. Brasília : M inistério da Saúde, 2000. 146p.

MONTEIRO, C.A. (Org.). Velhos e novos males da saúde no Brasil: a evolução do país e suas doenças. São Paulo: Hucitec, 1995. 359p.

ORGANIZAÇÃO PANAM ERICANA DE SAÚDE. A saúde no Brasil. Brasília, 1998. 82p.

PESQUISA NACIONAL SOBRE DEMOGRAFIA E SAÚDE 1996. Sociedade Civil Bem-Estar Familiar no Brasil. Programa de Demografia e Saúde. Rio de Janeiro, 1999. 182p.

RIBEIRO, D. O povo brasileiro: evolução e o sentido do Brasil. São Paulo : Companhia das Artes, 1995. $476 p$.

SANTOS, L.M.P., BATISTA, F.B.M., DINIZ, A.S. Epidemiologia de vitamina $A$ no Nordeste do Brasil. Boletin de la Oficina Sanitaria Panamericana, Washington DC, v.120, n.6, p.525-537, 1996a.
SANTOS, L.M.P., ASSIS, A.M.O., MARTINS, M.C., ARAÚJO, M.P.N., MORRIS, S.M., BARRETO, M.L. Situação alimentar e nutricional de pré-escolares no semi-árido da Bahia: Il hipovitaminose A. Revista de Saúde Pública, São Paulo, v.30, n.1, p.67-74, 1996b.

SANTOS, S.M.C. Fome e cidadania: a comunidade solidária e a participação em programas de nutrição em municípios baianos. Salvador, 2001. 335p. Tese (Doutorado em Administração Pública) - Escola de Administração, Universidade Federal da Bahia, 2001.

TRAD, L.A.B., BASTOS A.C.S. 0 impacto sociocultural do Programa Saúde da Família (PSF): uma proposta de avaliação, Cadernos de Saúde Publica, Rio de Janeiro, v.2, p.429-435, 1998.

VIANA, A., CASTRO, M.H.G. Brasil: a reforma do sistema de saúde. Santiago do Chile : Cepal. 1993. 83p. (Série Reformas de Política Pública).

WORLD HEALTH ORGANIZATION. Maternal anthropometry and pregnancy outcomes: a WHO collaborative study. Geneva, 1995. 98 p. (Supplement to volume 73).

WORLD HEALTH ORGANIZATION. Obesity: preventing and managing the global epidemic. Geneva, 1997. $276 p$.

Recebido para publicação em 13 de dezembro de 2000 e aceito em 12 de novembro DE 2001. 